\title{
Optimal traps in graphene
}

\author{
C. A. Downing, ${ }^{*}$ A. R. Pearce, and R. J. Churchill \\ School of Physics, University of Exeter, Stocker Road, Exeter EX4 4QL, United Kingdom
}

\author{
M. E. Portnoi ${ }^{\dagger}$ \\ School of Physics, University of Exeter, Stocker Road, Exeter EX4 4QL, United Kingdom \\ and International Institute of Physics, Universidade Federal do Rio Grande do Norte, 59078-400 Natal, RN, Brazil
}

(Received 8 May 2015; revised manuscript received 25 June 2015; published 1 October 2015)

\begin{abstract}
We transform the two-dimensional Dirac-Weyl equation, which governs the charge carriers in graphene, into a nonlinear first-order differential equation for scattering phase shift, using the so-called variable-phase method. This allows us to utilize the Levinson theorem, relating scattering phase shifts of a slow particle to its bound states, to find zero-energy bound states created electrostatically in realistic structures. These confined states are formed at critical potential strengths, which leads us to posit the use of "optimal traps" to combat the chiral tunneling found in graphene: this could be explored experimentally with an artificial network of point charges held above the graphene layer. We also discuss scattering on these states and find that the $s$ states create a dominant peak in the scattering cross section as the energy tends towards the Dirac point energy, suggesting a dominant contribution to the resistivity.
\end{abstract}

DOI: 10.1103/PhysRevB.92.165401

PACS number(s): 73.22.Pr, 73.21.La, 03.65.Ge, 03.65.Nk

\section{INTRODUCTION}

The electronic properties of the two-dimensional (2D) material graphene [1,2] are of great interest due to the quasirelativistic nature of its spectrum. Interesting transport effects such as chiral (Klein) tunneling [3-7], vacuum polarization [8], atomic collapse $[9,10]$, and the minimum conductivity at the Dirac point [11] have been widely discussed in the literature. The topic of elastic scattering in clean, low-temperature graphene, which can occur due to charged impurities, ripples, or strain fields, has been addressed by many authors [12-14].

However, despite its extraordinary properties there is a major obstacle stopping graphene from usurping silicon in the electronics industry, namely, its lack of a bandgap. This frustrates attempts to perform digital logic with graphene due to the difficulty in turning off the flow of chiral charge carriers, which always wish to conduct. Attempts at opening a gap in monolayer graphene have focused on cutting into nanoribbons [15], chemical functionalization [16], and strain engineering [17], which can unfortunately blunt the remarkable electronic properties that make graphene so attractive in the first place. Here we propose a method not to open a gap, but to switch off the chiral tunneling by considering zero-energy states, when the Fermi energy coincides with the Dirac points, such that fully confined states are predicted to be able to form due to the absence of pseudospin [18-20]. These states are also the most important factor when considering resonant scattering in graphene.

Despite the appearance of sophisticated experimental techniques for probing resonances and the modification of the density of states in the continuum [22], the search for fully confined (square-integrable) states remains a significant ongo-

\footnotetext{
*Now at Institut de Physique et Chimie des Matériaux de Strasbourg, Université de Strasbourg, CNRS UMR 7504, F-67034 Strasbourg, France.

†m.e.portnoi@exeter.ac.uk
}

ing task. Efficient manipulation of the Fermi level requires the presence of a back gate in close proximity to the graphene, which makes the numerous beautiful results stemming from the long-range behavior of the bare Coulomb potential [9] somewhat far from experimental reality, as the presence of image charges in the gate material (or screening effects) makes any realistic potential fall at large distances more rapidly than $1 / r$ [21]. It is important to emphasize that any fast-decaying potential cannot produce a bound state at nonzero energy [23]. Indeed, the asymptotics of the wave function is a Bessel function decaying at long distances as $r^{-1 / 2}$, and so one is led to consider zero-energy states instead. We also note that there is increasing interest in zero modes of the Dirac equation in the condensed matter community due to the possibilities of observing both the elusive Majorana fermions [24] or, indeed, fractionally charged excitations [25].

While low-energy resonant scattering in monolayer graphene has been extensively studied both theoretically $[9,23,26,27,29-34]$ and experimentally [35-38], the importance of fully confined zero-energy states in realistic structures has not been fully appreciated until recently [39]. In most of these papers, quasibound states, where only one wave-function component is confined or when the wave function is non-square-integrable, have been considered for resonant scattering. Previously, primarily fixed-depth circular wells $[23,26,40,41]$ or the Coulomb potential $[9,27]$ have been investigated, but in this paper we concentrate on smooth [20,42], short-range potentials which are defined by two parameters, characterizing both strength and spread. Notably, this is the only case where the commonly used single-valley approximation for graphene can be truly justified.

We study confined states and resonant scattering in graphene due to either scanning tunneling microscopy (STM) [43] of tip-induced potentials or due to some charge displaced out of the plane [44], with careful consideration of truly bound zero modes. The strength parameter can arise due to, for example, the size of the charge in the STM, while the spread parameter is linked to the distances from 
the scanning tunneling microscope tip to the graphene layer and back gate [20]. We investigate both the conditions required for a zero-energy bound state to form and the effect of such states on the energy dependence of the scattering cross section and resistivity contributions of resonant scatterers.

A complication that may arise from our proposed method of probing via STM is the appearance of a localized strain field [45] induced from the scanning tunneling microscope tip [46-48] in addition to the electrostatic potential we are interested in. Confinement due to the mechanically generated pseudomagnetic field is predicted to lead to an enhancement of the density of states in the deformed region [49-51]. However, the effect of tip-induced bumps, which arise via van der Waals forces, can be diminished by avoiding approaching the graphene membrane too closely, as the nature of the interaction is supershort range, for example, as in the Lennard-Jones potential.

To carry out our investigations of realistic, short-range (due to the necessity of a gate in all measurements) potentials we develop the ultrarelativistic analog of the variable-phase method (VPM) [52,53], which was found to be useful for tackling scattering problems governed by the Schrödinger equation in two dimensions [54-58], for use with the 2D DiracWeyl equation-allowing us to consider the charge carriers in graphene. The VPM was originally developed [59] for use with nonrelativistic wave equations in the 1930s as a neat tool to calculate physically relevant quantities directly, rather than having to extract them from the wave function, and has recently been developed for use with the Dirac-Weyl equation in quasi-1D problems $[60,61]$ due to the intense interest in graphene in the condensed matter community. Here we derive a first-order equation from which we can immediately find the scattering phase shift. This is advantageous, as important physical properties directly follow, such as the number of bound states (from the Levinson theorem) [55,56,62], the scattering and transport cross sections (from standard elastic scattering theory) [63], the number of states around a potential barrier (using the Friedel sum rule) [64], and the energy change due to the impurity interacting with neighboring electrons (the Fumi theorem) [65].

We have neglected effects due to rippling of or dislocations in the graphene membrane. We also do not discuss scattering by multiple electrostatic barriers $[66,67]$ or by magnetic barriers [68-70], but our method can be generalized to account for the presence of vector potentials.

The rest of this work is as follows. We outline the logic behind the VPM and give a detailed derivation of the phase equation for the Dirac-Weyl equation in Sec. II, which allows us to motivate the Levinson theorem. Section III is split into two parts. First, in Sec. III A we count bound states using the VPM, compare the results of the VPM against both tightbinding calculations on a finite flake and an exact solution of the Dirac equation, and posit a network of optimal traps which are able to hold and release carriers on demand. Second, in Sec. III B we apply elastic scattering theory in conjunction with the VPM, which allows us to investigate the influence of short-range scattering on some transport properties and unveil a remarkable formula relating phase shift to the nature of the potential well, valid at high energies. Finally, we summarize and discuss our results in Sec. IV. In Appendix A we show how quantum confinement of zero-energy states can occur for a quite general class of potential wells decaying at large distances as an inverse power law. In Appendix B we detail the key results for the simple case of scattering by a circular finitepotential well, in analogy with sharp, circular mass barriers, which have also been discussed in the literature [71].

\section{FORMALISM}

The 2D Dirac-Weyl Hamiltonian governing the low-energy charge carriers of graphene on a Dirac cone is [2]

$$
\hat{H}=v_{F} \boldsymbol{\sigma} \cdot \hat{\boldsymbol{p}}+U(r)+\sigma_{z} \Lambda,
$$

where $v_{F} \approx c / 300$ is the Fermi velocity of the Dirac particles, $\sigma=\left(\sigma_{x}, \sigma_{y}, \sigma_{z}\right)$ are the Pauli spin matrices, $U(r)$ is a central potential, and we include $\Lambda$ as the mass term for generality. A nonzero mass can arise due to chemical modifications, substrate-induced gaps [72] (e.g., on h-BN), or strain engineering [73]. We move into polar coordinates $(r, \theta)$ for circular symmetry and separate the variables via the following ansatz for the two-component spinor wave function

$$
\Psi(r, \theta)=\frac{e^{i m \theta}}{\sqrt{2 \pi}}\left(\begin{array}{c}
\chi_{A}(r) \\
i e^{i \theta} \chi_{B}(r)
\end{array}\right), \quad m=0, \pm 1, \pm 2, \ldots,
$$

where the subscripts $A$ and $B$ label the two sublattices of the graphene chicken-wire lattice. This choice of wave function leads to two coupled first-order differential equations for the radial wave-function components $\chi_{A, B}(r)$,

$$
\begin{gathered}
\left(\frac{d}{d r}+\frac{m+1}{r}\right) \chi_{B}=(k-V(r)-\Delta) \chi_{A}, \\
\left(-\frac{d}{d r}+\frac{m}{r}\right) \chi_{A}=(k-V(r)+\Delta) \chi_{B},
\end{gathered}
$$

with $V(r)=U(r) / \hbar v_{F}, \Delta=\Lambda / \hbar v_{F}$, and $k=E / \hbar v_{F}$, where $E$ is the eigenenergy. Rearranging Eq. (3) into a secondorder differential equation for a single radial wave-function component $\chi_{A}(r)$, we obtain for the massless case

$$
\begin{aligned}
& \frac{d^{2}}{d r^{2}} \chi_{A}(r)+\left(\frac{1}{r}+\frac{1}{k-V(r)} \frac{d V(r)}{d r}\right) \frac{d}{d r} \chi_{A}(r) \\
& +\left((k-V(r))^{2}-\frac{m}{r} \frac{1}{k-V(r)} \frac{d V(r)}{d r}-\frac{m^{2}}{r^{2}}\right) \chi_{A}(r)=0 .
\end{aligned}
$$

We consider potentials of the form $V(r \rightarrow \infty)=0$ such that at large distances Eq. (4) reduces to [74]

$$
\frac{d^{2}}{d r^{2}} \chi_{A}(r)+\left(\frac{1}{r}\right) \frac{d}{d r} \chi_{A}(r)+\left(k^{2}-\frac{m^{2}}{r^{2}}\right) \chi_{A}(r)=0,
$$

which is the Bessel equation with the well-known solution $a_{m} J_{m}(k r)-b_{m} N_{m}(k r)$ or, equivalently,

$$
\chi_{A}(r)=A_{m}\left[J_{m}(k r) \cos \left(\delta_{m}\right)-N_{m}(k r) \sin \left(\delta_{m}\right)\right],
$$

where $J_{m}(k r)$ and $N_{m}(k r)$ are the Bessel functions of the first and second kinds, respectively, and $\delta_{m}=\arctan \left(b_{m} / a_{m}\right)$ is the scattering phase shift, arising from the difference in phase of the wave function at $r \rightarrow \infty$ compared to the free particle case. 
We now implement the VPM by treating the constants $A_{m}$ and $\delta_{m}$ as functions of the radial coordinate $r$, such that

$\chi_{A}(r)=A_{m}(r)\left[J_{m}(k r) \cos \left(\delta_{m}(r)\right)-N_{m}(k r) \sin \left(\delta_{m}(r)\right)\right]$,

where $A_{m}(r)$ is called the amplitude function and the phase function $\delta_{m}$ is the phase shift arising from a potential cutoff at a distance $r$. To completely define these newly introduced functions $A_{m}(r)$ and $\delta_{m}$ we make the standard [52,53] ansatz for the first derivative of $\chi_{A}(r)$ with respect to $r$,

$\chi_{A}^{\prime}(r)=A_{m}(r)\left[J_{m}^{\prime}(k r) \cos \left(\delta_{m}(r)\right)-N_{m}^{\prime}(k r) \sin \left(\delta_{m}(r)\right)\right]$,

where the prime symbol denotes differentiation with respect to $r$. Now, setting the full derivative of Eq. (7) equal to Eq. (8) yields the following useful condition:

$\frac{A_{m}^{\prime}(r)}{A_{m}(r)}=\delta_{m}^{\prime}(r) \frac{J_{m}(k r) \sin \left(\delta_{m}(r)\right)+N_{m}(k r) \cos \left(\delta_{m}(r)\right)}{J_{m}(k r) \cos \left(\delta_{m}(r)\right)-N_{m}(k r) \sin \left(\delta_{m}(r)\right)}$.

Upon substituting Eq. (7) and Eq. (8) into the lower coupled Eq. (3b) we naturally find that the lower radial wave-function component $\chi_{B}(r)$ is

$$
\begin{aligned}
\chi_{B}(r)= & \frac{A_{m}(r)}{k-V(r)}\left[\left(-J_{m}^{\prime}(k r)+\frac{m}{r} J_{m}(k r)\right) \cos \left(\delta_{m}(r)\right)\right. \\
& \left.-\left(-N_{m}^{\prime}(k r)+\frac{m}{r} N_{m}(k r)\right) \sin \left(\delta_{m}(r)\right)\right] .
\end{aligned}
$$

We can now utilize the upper coupled Eq. (3a): upon substituting in Eq. (7) and Eq. (10) and eliminating the amplitude function $A_{m}(r)$ via the application of the condition, Eq. (9), we obtain the first-order differential equation

$$
\begin{aligned}
\frac{d}{d r} \delta_{m}(r)= & \frac{\pi r}{2} p(r)\left[\frac{1}{k-V(r)} \frac{d V(r)}{d r}\left(q(r)-\frac{m}{r} p(r)\right)\right. \\
& \left.+\left(V(r)^{2}-2 k V(r)\right) p(r)\right], \\
p(r)= & J_{m}(k r) \cos \left(\delta_{m}(r)\right)-N_{m}(k r) \sin \left(\delta_{m}(r)\right), \\
q(r)= & J_{m}^{\prime}(k r) \cos \left(\delta_{m}(r)\right)-N_{m}^{\prime}(k r) \sin \left(\delta_{m}(r)\right),
\end{aligned}
$$

where we have introduced the auxiliary functions $p(r)$ and $q(r)$ and, in addition have used the Wronskian of the Bessel functions $W\left\{J_{m}(x), N_{m}(x)\right\}=J_{m}(x) N_{m}^{\prime}(x)-N_{m}(x) J_{m}^{\prime}(x)=$ $-2 /(\pi x)$ to simplify the final expression [75]. Equation (11) is the so-called phase equation and is subject to the initial condition $\delta_{m}(0)=0$, as follows from being in the free particle limit. We can see from Eq. (11) how the potential $V(r)$ gradually accumulates the desired phase shift starting from $\delta_{m}(0)=0$ and finishing with the total phase shift of the scattering problem, given by

$$
\delta_{m}=\lim _{r \rightarrow \infty} \delta_{m}(r) .
$$

This condition ensures that the phase shift is uniquely defined, avoiding the ambiguity of $\pi$ that appears in other methods [63]. When investigating bound states in the massless case we can only consider zero-energy states, where the Neumann function is divergent and so the following condition is implied:

$$
\delta_{m}=n \pi, \quad n=1,2,3 \ldots
$$

Equation (13) is related to the Levinson's theorem for massless 2D Dirac particles, which states a relation between the phase shift at zero momentum and the number of bound states.
Please note when considering the massive 2D Dirac particles, as found in gapped graphene, which allow bound states at finite energy, that an equation analogous to the phase equation, Eq. (11), can be derived for treating confined states,

$$
\begin{aligned}
\frac{d}{d r} \eta_{m}(r)= & -r f(r)\left[\frac{1}{k-V(r)} \frac{d V(r)}{d r}\left(g(r)-\frac{m}{r} f(r)\right)\right. \\
& \left.+\left(V(r)^{2}-2 k V(r)\right) f(r)\right], \\
f(r)= & I_{m}(\kappa r) \cos \left(\eta_{m}(r)\right)-K_{m}(\kappa r) \sin \left(\eta_{m}(r)\right), \\
g(r)= & I_{m}^{\prime}(\kappa r) \cos \left(\eta_{m}(r)\right)-K_{m}^{\prime}(\kappa r) \sin \left(\eta_{m}(r)\right),
\end{aligned}
$$

where $I_{m}(\kappa r)$ and $K_{m}(\kappa r)$ are the modified Bessel and Neumann functions, respectively, and the effective wave vector $\kappa=\left(\Delta^{2}-k^{2}\right)^{1 / 2}$. Equation (14) has been simplified [75] by noting that the Wronskian of the modified Bessel functions $W\left\{I_{m}(x), K_{m}(x)\right\}=I_{m}(x) K_{m}^{\prime}(x)-K_{m}(x) I_{m}^{\prime}(x)=$ $-1 / x$. Notably, Eq. (14) is also relevant for considerations of the surface states on $3 \mathrm{D}$ topological insulators such as $\mathrm{Bi}_{2} \mathrm{Te}_{3}$, where in this case the mass term arises from the exchange energy from a magnetic insulator [76]. When considering bound states we note that the modified Bessel function of the first kind is divergent and so the following condition is implied,

$$
\eta_{m}=\left(n-\frac{1}{2}\right) \pi, \quad n=1,2,3, \ldots,
$$

which is a representation of the Levinson theorem for the massive 2D Dirac equation in a central potential, studied previously using the Green's function method [77] and via a utilization of the generalized Sturm-Liouville theorem [78].

\section{RESULTS}

\section{A. Counting bound states}

We now check that we can reproduce known results by solving the phase equation, Eq. (11), describing the massless charge carriers of graphene, for the case of zero-energy states formed in a Lorentzian potential, $V(r)=-V_{0} /\left(1+(r / d)^{2}\right)$, an analytically solved problem [20]. In this case the condition for bound states when $m \geqslant 0$ is $V_{0} d=2(N+m)$, where $N=$ $1,2, \ldots$ is a positive integer. Thus, when solving Eq. (11), the phase equation for massless Dirac particles, for $k \rightarrow 0$ we should see that the threshold value of $V_{0} d$ is reached just before the first step in the phase shift-versus-potential strength plot; as $V_{0} d$ is increased more confined zero-energy states appear. This is exactly what we find in Fig. 1(a). We also note that the sign of $V_{0}$ is irrelevant for the creation of zero-energy confined states since a well for an electron is a hill for a hole, and vice versa, thus Fig. 1 has mirror symmetry about the $V_{0} d=0$ axis. At the Dirac point, when the density of states vanishes yet the conductivity remains finite, these zero-energy states should be important, as we see later.

While screening affects only the strength of the Coulomb potential, and not its characteristic decay law [14], due to the quasirelativistic nature of the carriers in graphene, a cutoff is necessary at the origin and the presence of an image charge in the metallic back gate will lead to a dipole-like $1 / r^{3}$ decay at large distances, thus a more realistic choice of model potential 

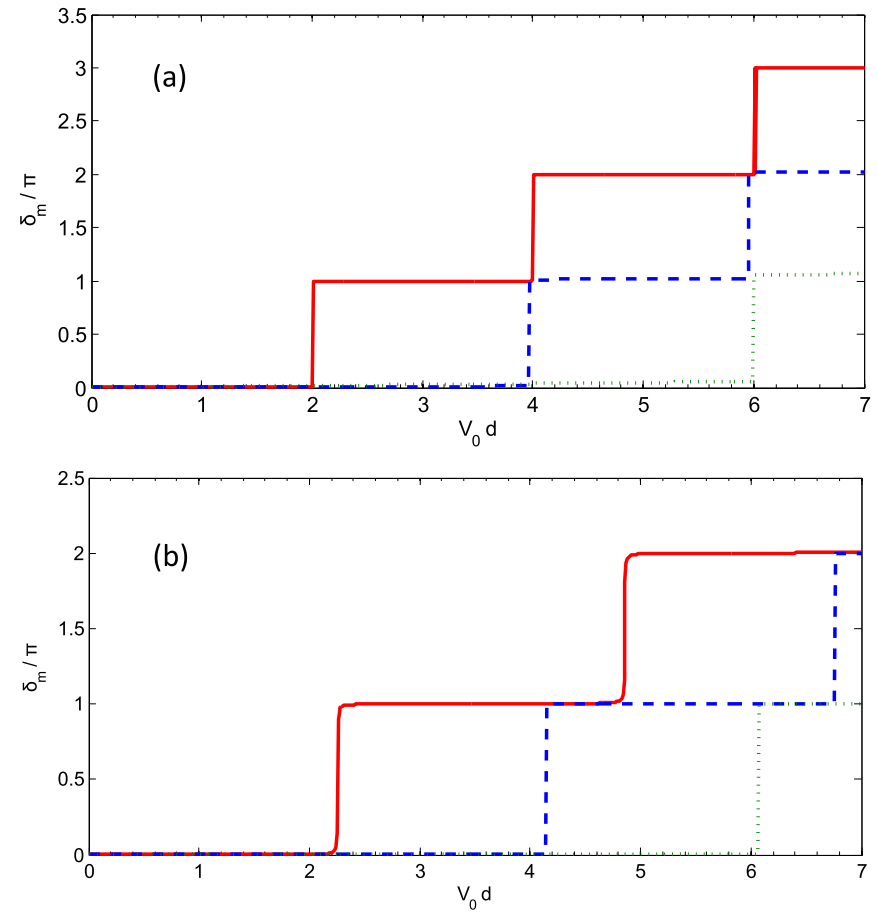

FIG. 1. (Color online) Plots of scaled phase shifts $\delta_{m} / \pi$ versus potential strength $V_{0} d$ for massless Dirac particles, of energy tending towards 0 , incident on (a) the Lorentzian potential and (b) the model potential of Eq. (16) with the realistic $1 / r^{3}$ decay. We show results for angular momentum $m=0,1$, and 2, corresponding to the solid (red) line, dashed (blue) line, and dotted (green) line, respectively.

is

$$
V(r)=\frac{-V_{0}}{1+(r / d)^{3}} .
$$

In Fig. 1(b) we investigate confined states in a trapping potential given by Eq. (16) and again see a characteristic threshold in $V_{0} d$ for the emergence of the first bound state, followed by the signature staircase behavior of confined zero-energy states. Of course, compared to the exactly solvable Lorentzian potential, with its accidental degeneracies, the staircase does not share the same beautiful symmetries and the condition for full-confined states can be approximated by $V_{0} d \approx 2.63 N+1.89 m-0.34$, which is valid for the range of parameters used in Fig. 1(b). However, we can now predict for realistic graphene flakes, where we would expect charged impurities to cause potentials similar to the type of Eq. (16), that there is the possibility of the appearance of fully confined zero-energy states, which are clearly not an artifact of a particular analytically solvable model potential. Small perturbations away from the condition for zero-energy states lead to states with complex energies, which can be ascribed to a lifetime which is increasingly long for states with a higher angular momentum [5] and in smoother traps [79].

Note, as we show in Appendix A by considering a general class of potential wells regular at the origin and decaying like some power law (which is faster than the Coulomb potential), that the toy model confinement potential one chooses is not crucial for the actual existence of zero-energy bound states but is important for knowing where one should search for them in terms of potential strength. Thus we have shown that it is possible to create electrostatic traps holding electrons (or holes) in the regime where the Fermi level is close to the Dirac-point energy. Then it should be possible to release these trapped quasiparticles into the system by a small adjustment of the trapping potential strength and spread, which will result in the sought-after on/off behavior.

Such states should be able to be detected in scanning probe microscopy experiments, as proposed in Ref. [20], where smoothly changing either the charge on the scanning probe microscope tips or their distance above the graphene plane, and continuously holding the Fermi level at the Dirac point using the back gate, should be sufficient to see confined zero modes. A network of sparse scanning probe microscope tips of radius $R_{\text {tip }}$, separated in a square grid defined by an intertip separation $s$, all held at a distance $h_{2}$ above a metallic back gate and $h_{2}-h_{1}$ above the graphene plane, gives a similarly behaved potential to Eq. (16), but in a more complicated form, which can be obtained via the method of images,

$$
\begin{aligned}
U(r)= & \frac{e Q_{\mathrm{tip}}}{4 \pi \epsilon_{0} \epsilon_{r}} f(r) \\
f(r)= & \sum_{j, k=-n_{1}}^{n_{2}}\left((x+j s)^{2}+(y+k s)^{2}+\left(h_{2}-h_{1}\right)^{2}\right)^{-1 / 2} \\
& -\left((x+j s)^{2}+(y+k s)^{2}+\left(h_{2}+h_{1}\right)^{2}\right)^{-1 / 2}
\end{aligned}
$$

Fitting Eq. (16) to Eq. (17) by matching the functions at both their maximum and their half-maximum values, one finds that the tip voltage $V_{\text {tip }}$ at which one would see such zero modes is

$$
V_{\text {tip }}=\frac{\left(V_{0} d\right)_{N, m}}{f(0) d} \frac{\hbar v_{F}}{e R_{\text {tip }}},
$$

subject to the constraint $2 f(d)=f(0)$, where we have seen from Fig. 1(b) the dimensionless parameter $\left(V_{0} d\right)_{N, m \neq 0}=$ $4.16,6.06,6.79, \ldots$, such that we are dealing with [80] tip voltages of the order of tens of millivolts: $V_{\text {tip }}=20 \mathrm{mV}, 29 \mathrm{mV}$, $33 \mathrm{mV}$, and so on. Thus at particular voltages, optimal traps are created which can release conducting particles on demand when the voltage differs from the optimal value.

The existence of confined states opens up the possibility of Coulomb-blockade-type physics in graphene. Indeed, the quantum dots created with careful adjustment of the key parameters can be be seen to be "optimal traps." An estimate of the charging energy of the optimal trap, using a simple disk capacitor model, shows a charging energy of the order of milli-electron volts; thus, such effects could be seen even at room temperature. The effect arises due to the tightly confined nature of the wave function in optimal traps, which leads to a low capacitance and a significant charging energy, which is negligible for deconfined states.

We also show in Fig. 2 that the zero modes of the Dirac equation in the potential, Eq. (16), as predicted in the continuum model, are indeed present as shown via tight-binding calculations $[81,82]$. It is found that the wave functions have a ringlike structure, which ensures avoidance of any Klein tunneling effects, i.e., the states are zero-energy vortices with $m \neq 0$. It is most noticeable how by adjusting the parameter $V_{0} d$ one can go from a tightly confined state to a state with a widely spread probability density. The $60^{\circ}$ 

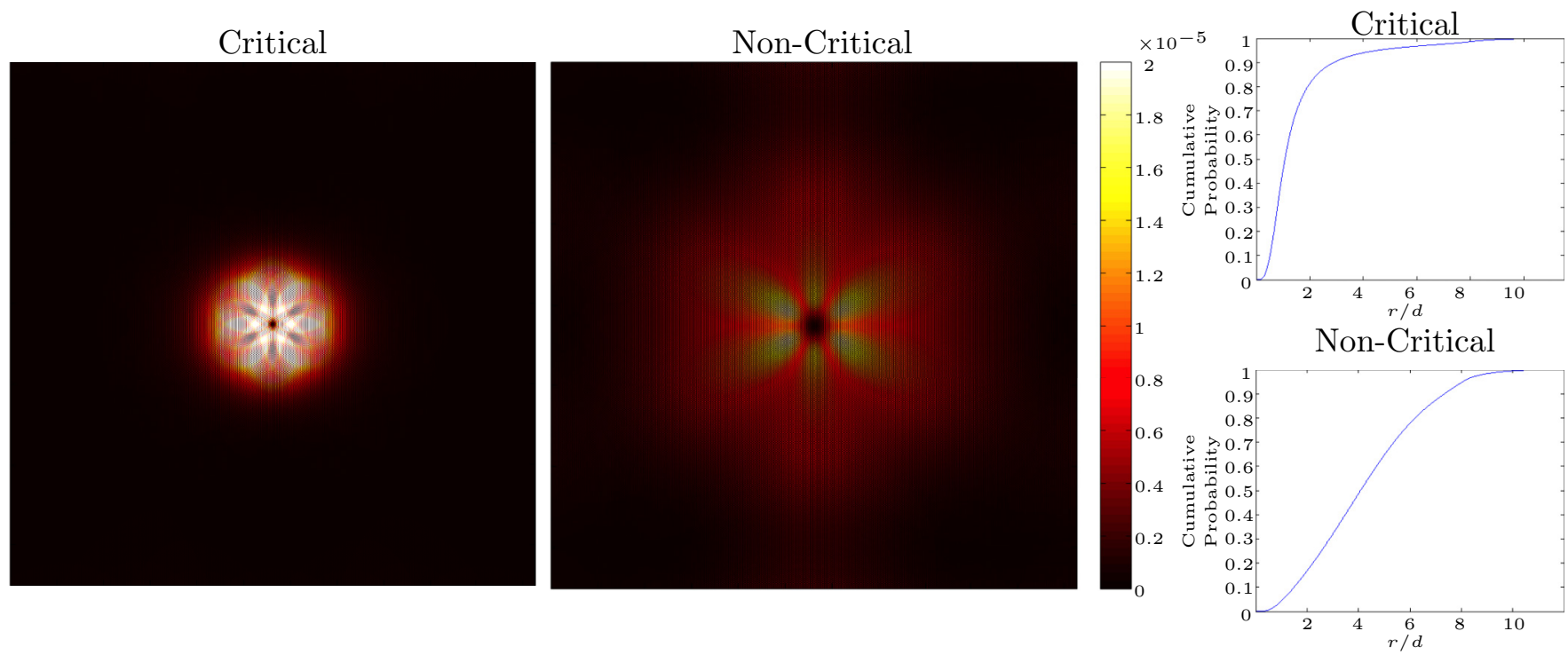

FIG. 2. (Color online) Probability density plots of near-zero-energy states in the model confining potential given by Eq. (16), resulting from tight-binding calculations for a finite flake. We show example critical $V_{0} d=4.10$ (left) and noncritical $V_{0} d=2.60$ (middle) states, as well as the associated cumulative probability plots (right).

rotation symmetry of the numerical results clearly shown in Fig. 2 is changed to a $120^{\circ}$ symmetry when the center of the potential is chosen on one of the carbon atoms instead of the center of the elementary cell. We have also checked that the zero modes are robust to the shape of the graphene flake by investigating triangle and hexagon geometries, with both zigzag and armchair boundaries. In fact, the bound state on finite flakes seem to be even more robust than in the infinite, ideal system, as all effects violating pseudospin conservation at nonzero energy required by the Weyl equation help to bind the particle. Nevertheless, our numerical experiments show that a modest change in potential from the optimal value lead to complete bound-state dissociation.

\section{B. Resonant scattering}

As mentioned previously, once the scattering phase shift is known a number of other useful physical properties can be quickly calculated. The partial cross section $\zeta_{m}$ is

$$
k \zeta_{m}=\sin ^{2}\left(\delta_{m}\right),
$$

while the total scattering cross section $\zeta$, a measure of the 1D area felt by incoming particles, and the transport cross section $\zeta_{T}$ easily follow from the scattering phase shifts via

$$
\zeta=\frac{4}{k} \sum_{m=-\infty}^{\infty} \sin ^{2}\left(\delta_{m}\right), \quad \zeta_{T}=\frac{2}{k} \sum_{m=-\infty}^{\infty} \sin ^{2}\left(\delta_{m+1}-\delta_{m}\right),
$$

and we note that for low-energy scattering we can take the near$s$-wave approximation, i.e., only small values of $m$ need to be considered, as Eq. (20) is derived from partial-wave expansions where terms with high $m$ are negligible when $k \rightarrow 0$. Detailed derivations of Eqs. (19) and (20) via the 2D elastic scattering theory adapted for Dirac fermions have been given by Novikov in Ref. [27] and are exactly the same as in the nonrelativistic 2D case [28].
The energy dependence of the scattering cross section has previously been considered for a square well $[23,26]$ and we outline a thorough solution to this problem in Appendix B. We now revisit the problem with a smooth, short-range potential given by Eq. (16), which is relevant for gated structures or for hypercritical charges. Plots of the dimensionless partial cross section $k \zeta_{m}$ are shown for the case of noncritical [Fig. 3(a)] and critical [Fig. 3(b)] parameters of the potential, Eq. (16). We find that, in contrast to the noncritical case, at the critical potential strength the $m=0$ non-square-integrable state does not go to 0 in $k \zeta_{m}$ as quickly as $k$ tends towards 0 , and thus the partial cross section $\zeta_{m}$ is divergent as the energy tends towards 0 . This is because $m=0$ is a resonant state with a non-normalizable wave function (and a nonzero probability density at the origin, $r=0$ ) and so such a particle has an enhanced likelihood of being scattered. Of course, this behavior also manifests in calculations of the transport scattering cross section via Eq. (20). The problem of scattering involving ultrashort-range midgap states, which may arise due to vacancies or cracks, is closely related.[83]

We also note, in striking contrast to the case of nonrelativistic particles, that at high energies the phase shift $\delta_{\infty}$ is nonzero, which is similar to the 3D relativistic case [84]. Remarkably, $\delta_{\infty}$ is also angular momentum independent, and we find from Eq. (11) its explicit form,

$$
\delta_{\infty}=-\int_{0}^{\infty} V(r) \mathrm{d} r
$$

such that for the considered potential given by Eq. (16) the dimensionless partial scattering cross section in the highenergy limit is given by $k \zeta_{m} \rightarrow \sin ^{2}\left(2 \pi / 3 \sqrt{3} V_{0} d\right)$. This limit is already reached for the upper values of $k d$ used in Fig. 3. Thus, the transport cross section $\zeta_{T}$ indeed vanishes in the large- $k$ limit.

The smoking gun of confined zero modes in the laboratory could be via their contribution to resistivity, which in the 

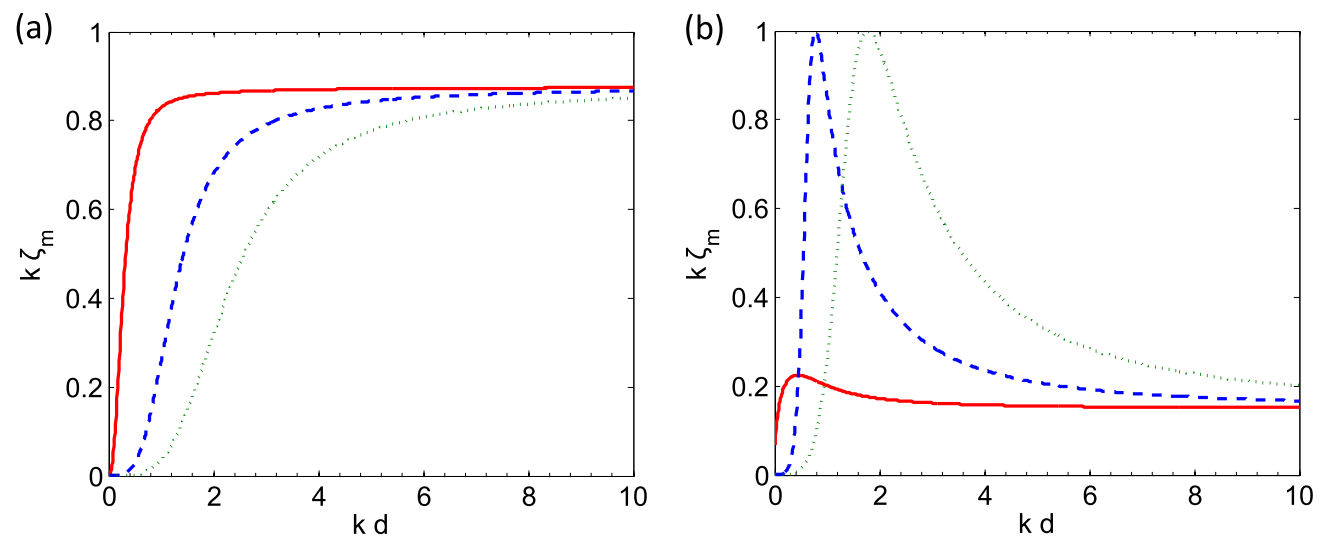

FIG. 3. (Color online) Plot of the dimensionless partial scattering cross section $k \zeta_{m}$ as a function of the scaled energy $k$ for massless Dirac particles incident on the model potential with $1 / r^{3}$ decay given by Eq. (18), with example (a) noncritical potential strength $V_{0} d=1.00$ and (b) critical potential strength $V_{0} d=2.27$. We show results for $m=0,1$, and 2, corresponding to the solid (red) line, dashed (blue) line, and dotted (green) line, respectively.

semiclassical Boltzmann theory can be expressed as [26]

$$
\rho=\frac{h}{4 e^{2}} \frac{2 n_{s}}{\pi n_{e}} k_{F} \zeta_{T}
$$

where $n_{s}$ is the density of scatterers and the electron density $n_{e}=k_{F}^{2} / \pi$ in the Fermi limit. We show in Fig. 4 the behavior of resistivity at small $k_{F} d$. The presence of confined zero modes leads to a divergence in resistivity, $\rho \rightarrow \infty$, at energies tending towards 0 in the critical case only. It should be possible to utilize this consequent drastic suppression of mobility to create an off state in graphene, which can be explored artificially with a series of point gates held above the graphene monolayer. In the noncritical cases, we find that as $k_{F} \rightarrow 0$ resistivity saturates to a constant.

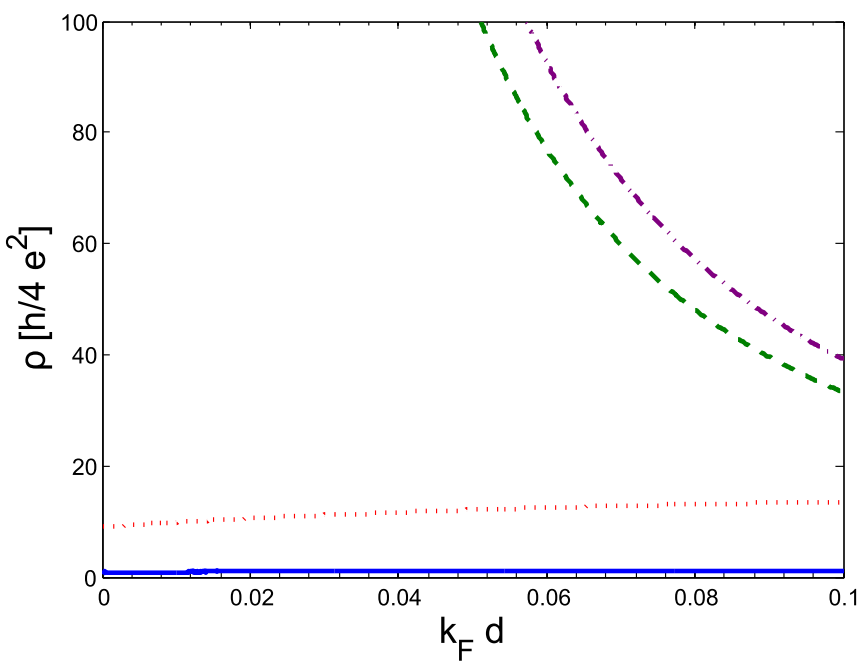

FIG. 4. (Color online) Plot of the resistivity $\rho$, measured in units of $h / 4 e^{2}$, as a function of the energy $k_{F} d$ for massless Dirac particles scattering on the model potential with $1 / r^{3}$ decay. We show results for the uncritical cases $V_{0} d=1.00$ and 3.00 , corresponding to the dotted (red) line and solid (blue) line, respectively, and the critical cases $V_{0} d=2.27$ and 4.87, corresponding to the dashed (green) line and dot-dashed (purple) line, respectively. We set $2 n_{s} d^{2}=1$.

\section{DISCUSSION}

We have derived the phase equations for the 2D Dirac equation using the VPM, suitable for use straightaway in scattering calculations concerning Dirac-Weyl materials such as graphene. In doing so, we also provide both a numerical (heuristic) proof of the Levinson theorem for massless 2D particles and a simple relation for phase shift at high energies. These phase equations are of first order, and so relatively undemanding computationally, and have solutions in terms of scattering phase shifts, thus other desired scattering properties readily follow. Applying this method to count fully confined states in graphene, we reproduce a known exact result and go on to investigate a more physically relevant potential, finding that at certain potential strengths and spreads zero-energy bound states are likely to form, a fact that can be exploited with STM tip potentials.

It should also be mentioned that the existing experiments [85] on atomic clusters on a graphene surface can arguably be explained by the considered zero-energy states without involving the atomic collapse picture. These experiments deal with back-gated graphene, which precludes the use of a long-range Coulomb potential. The measured wave-function density has a pronounced ringlike structure on a scale of many lattice constants, instead of a sharp peak at the atomic scale as expected from "fall-into-the-center" physics. Moreover, the observed features in the density of states are very close to zero energy (in fact, on both sides of the Dirac point) instead of being a few electron volts away from this energy as expected for a collapsed state.

We have also calculated the energy dependence of the scattering cross section, finding a major distinction for the $m=0$ mode for critical potential strengths. In this special case, we predict a dominant peak in scattering cross section at zero energy, suggesting a high probability of being scattered, which can be explored experimentally via scanning probe microscopy. In an experiment with a series of point gates above the graphene layer, one may be able to use artificial resonant scattering to switch off the chiral tunneling effect found in monolayer graphene by greatly reducing the mobility of carriers. 
Note Added in Proof: After submission of our manuscript, we became aware of a new experiment [86] reporting confinement of quasibound or so-called whispering-gallery modes in graphene via a scanning tunneling probe. The highly tunable setup described, wherein both the electron wavelength and the tip-induced circular $p n$ junction radius are controlled independently, suggests that the arguably more exotic truly bound states (zero-energy vortices) discussed in this paper can soon be thoroughly probed. Notably, the setup is similar to the one discussed here and a gate is already present, although its effect is not discussed.

\section{ACKNOWLEDGMENTS}

This work was supported by the EPSRC (CAD) and the EU FP7 ITN NOTEDEV (Grant No. FP7-607521) and FP7 IRSES projects CANTOR (Grant No. FP7-612285), QOCaN (Grant No. FP7-316432), and InterNoM (Grant No. FP7-612624).

\section{APPENDIX A: BOUND STATES IN POTENTIAL WELLS DECAYING AS A POWER LAW}

Let us examine the condition for bound states to form in regularized potential wells decaying asymptotically as a power law governed by a parameter $p>1$, namely,

$$
V(r)=-V_{0}\left\{\begin{array}{lll}
1 & \text { if } & r \leqslant R, \\
\left(\frac{R}{r}\right)^{p} & \text { if } & r>R,
\end{array}\right.
$$

such that the wave-function components inside the well are simply Bessel functions and outside the well are the function $r^{-p / 2}$ product Bessel functions in a new variable, $\xi=V_{0} R(R / r)^{(p-1)} /(p-1)$. Bound zero-energy states can never arise for $m=0,-1$, due to the requirement of some rotation to combat the Klein tunneling phenomenon [20]. Furthermore, some other negative angular momentum states $m_{e} \leqslant m \leqslant-1$ are excluded, depending on the power of the decay, for $p>-2 m_{e}$. Thus, as one considers increasingly short-range interactions, one will find a wider band of "missing" nonpositive integer angular momentum states cumulating in $m_{e}$. The potential parameters corresponding to the squareintegrable solutions can be found from the determinant of the matrix

$$
\left|\begin{array}{cc}
J_{|m|}\left(V_{0} R\right) & J_{|m+1|}\left(V_{0} R\right) \\
J_{|\alpha|}\left(\frac{V_{0} R}{p-1}\right) & J_{|\alpha-1|}\left(\frac{V_{0} R}{p-1}\right)
\end{array}\right|=0, \quad \alpha=\frac{m+p / 2}{p-1},
$$

which can be solved by the usual root-finding methods for the values of $V_{0} R$ which are able to support confined modes. In the limit of large $V_{0} R \gg 1$, Eq. (A2) can be rewritten in terms of elementary functions,

$\left|\begin{array}{lr}\cos \left(V_{0} R-\frac{\pi}{2}|m|-\frac{\pi}{4}\right) & \cos \left(V_{0} R-\frac{\pi}{2}|m+1|-\frac{\pi}{4}\right) \\ \cos \left(\frac{V_{0} R}{p-1}-\frac{\pi}{2}|\alpha-1|-\frac{\pi}{4}\right) & \cos \left(\frac{V_{0} R}{p-1}-\frac{\pi}{2}|\alpha|-\frac{\pi}{4}\right)\end{array}\right|=0$,

and the most symmetric case, $p=2$, is equivalent to $V_{0} R=$ $\frac{\pi}{4}(|m|+|m+1|+2 n+1)$, where $n$ is an integer.

While this somewhat kinky class of potential is not as realistic as the smooth potentials considered in the text, it quantitatively demonstrates how bound zero-energy states are realizable in many situations for fast-decaying potentials. As expected, a separate analysis for the regularized Coulomb potential $(p=1)$ yields no possibility of bound zero-energy states. The corresponding transcendental equation cannot be satisfied for any real value of $V_{0} R$.

\section{APPENDIX B: SCATTERING BY A CIRCULAR FINITE-POTENTIAL WELL}

Let us consider the example of a circular finite-potential well [23] given by $V(r)=-V_{0} \Theta(d-r)$, where $\Theta(z)$ is the Heaviside step function, which allows us to obtain the following solutions of Eq. (4): $\chi_{A}(r)=A_{m} J_{m}\left(\left(k+V_{0}\right) r\right)$ inside the well and $\chi_{A}(r)=B_{m}\left[J_{m}(k r) \cos \left(\delta_{m}\right)-N_{m}(k r) \sin \left(\delta_{m}\right)\right]$ outside the well. Upon finding $\chi_{B}(r)$ from Eq. (3b) and matching both wave-function components at the boundary $r=d$, we obtain the following expression for the tangent of the phase shift:

$$
\begin{aligned}
& \tan \left(\delta_{m}\right) \\
& \quad=\frac{J_{m}\left(\left(k+V_{0}\right) d\right) J_{m+1}(k d)-J_{m+1}\left(\left(k+V_{0}\right) d\right) J_{m}(k d)}{J_{m}\left(\left(k+V_{0}\right) d\right) N_{m+1}(k d)-J_{m+1}\left(\left(k+V_{0}\right) d\right) N_{m}(k d)} .
\end{aligned}
$$

It can be seen that resonances in the scattering cross section do exist at certain energies, as can be seen by substituting Eq. (B1) into Eq. (19) or into the more convenient form $k \zeta_{m}=1 /(1+$ $\left.\tan \left(\delta_{m}\right)^{-2}\right)$. At energies tending to the Dirac-point energy $k \zeta_{m}$ and, more importantly, the true partial cross section $\zeta_{m}$ go to 0 . At high energies we find the expected constant value, given by $k \zeta_{m} \rightarrow \sin ^{2}\left(V_{0} a\right)$, as follows from Eq. (21). Both of these features are shown in Fig. 5(a). One can find the most prominent resonances of the partial cross section for this problem, defined by $k \zeta_{m}=1$, which can only occur for some specific potential strengths, which satisfy the condition $J_{m}\left(\left(k+V_{0}\right) d\right) N_{m+1}(k d)=J_{m+1}\left(\left(k+V_{0}\right) d\right) N_{m}(k d)$. If there is a solution to this equation, it is straightforward to find the resonance energy, which is the global maximum solution of $\frac{d}{d k} \zeta_{m}=0$.

In the $s$-wave approximation, one can see by using Eq. (B1) in conjunction with Eq. (20) that the contribution to resistivity, calculated via Eq. (22), is given by [26]

$$
\rho \approx \frac{h}{4 e^{2}} n_{s} d^{2}
$$

which, of course, can be neglected when the concentration of scatterers and/or the scattering radius $d$ is small. However, this analysis neglects the possible existence of confined zero modes, which can be determined by the condition $J_{m}\left(V_{0} d\right)=0$. The effect of the extended $m=0$ state (which occurs at the zeros of the zeroth Bessel function, $V_{0} d=2.40,5.52, \ldots$ ) is to see the quantity $\tan \left(\delta_{m}\right)$ approach 0 slowly (logarithmically) as we approach the Dirac-point energy, such that $k \zeta_{0}$ effectively tends to a constant, producing a "superresonance" at zero energy in the pure partial cross section $\zeta_{0}$, as displayed in Fig. 5(b). This leads to a noticeable contribution to the resistivity [26],

$$
\rho \approx \frac{h}{4 e^{2}} \frac{n_{s}}{n_{e}} \frac{1}{\ln ^{2}(k d)},
$$

which is clearly more dominant than that found in Eq. (B2). 
(a)

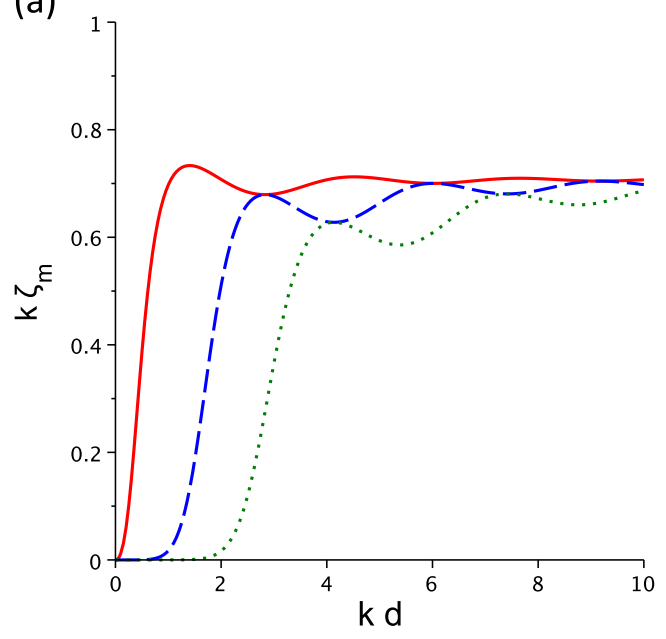

(b)

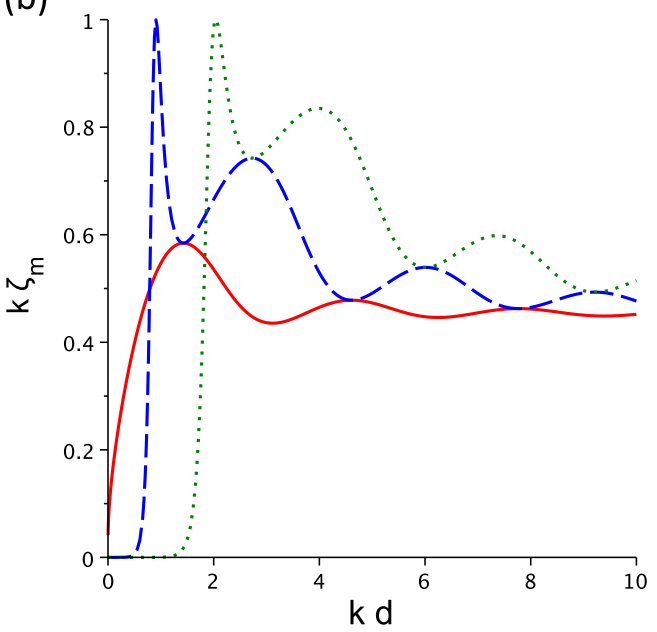

FIG. 5. (Color online) Plot of the dimensionless partial scattering cross section $k \zeta_{m}$ as a function of the scaled energy $k$ for massless Dirac particles incident on the circular finite-potential well, with (a) noncritical potential strength $V_{0} d=1$ and (b) critical potential strength $V_{0} d=2.40$. We show results for $m=0,1$, and 2, corresponding to the solid (red) line, dashed (blue) line, and dotted (green) line, respectively.

We would like to mention an intriguing experiment [87] which has recently mapped the total cross section for a circular gated region in a self-assembled molecular-graphene hybrid and indeed found resonant peaks. These resonances can be thought of as whispering gallery modes, which have just been probed in graphene [86] using the tip of a scanning tunneling microscope, which are directly related to the zero-energy states discussed in this work.
[1] K. S. Novoselov, A. K. Geim, S. V. Morozov, D. Jiang, Y. Zhang, S. V. Dubonos, I. V. Gregorieva, and A. A. Firsov, Science 306, 666 (2004).

[2] D. S. L. Abergel, V. Apalkov, J. Berashevich, K. Ziegler, and T. Chakraborty, Adv. Phys. 59, 261 (2010).

[3] O. Klein, Z. Phys. 53, 157 (1929).

[4] M. I. Katsnelson, K. S. Novoselov, and A. K. Geim, Nat. Phys. 2, 620 (2006).

[5] P. Hewageegana and V. Apalkov, Phys. Rev. B 77, 245426 (2008).

[6] A. F. Young and P. Kim, Nat. Phys. 5, 222 (2009).

[7] T. Tudorovskiy, K. J. A. Reijnders, and M. I. Katsnelson, Phys. Scripta, T 146, 014010 (2012).

[8] A. V. Shytov, M. I. Katsnelson, and L. S. Levitov, Phys. Rev. Lett. 99, 236801 (2007).

[9] A. V. Shytov, M. I. Katsnelson, and L. S. Levitov, Phys. Rev. Lett. 99, 246802 (2007); A. V. Shytov, M. S. Rudner, N. Gu, M. I. Katsnelson, and L. S. Levitov, Solid State Commun. 149, 1087 (2009).

[10] C. A. Downing and M. E. Portnoi, Phys. Rev. A 90, 052116 (2014).

[11] K. S. Novoselov, A. K. Geim, S. V. Morovoz, D. Jiang, M. I. Katsnelson, I. V. Gregorieva, S. V. Dubonos, and A. A. Firsov, Nature (London) 438, 197 (2005).

[12] S. Adam, E. H. Hwang, E. Rossi, and S. Das Sarma, Solid State Commun. 149, 1072 (2009)

[13] N. M. R. Peres, Rev. Mod. Phys. 82, 2673 (2010).

[14] S. Das Sarma, S. Adam, E. H. Hwang, and E. Rossi, Rev. Mod. Phys. 83, 407 (2011).

[15] L. Brey and H. A. Fertig, Phys. Rev. B 73, 235411 (2006).
[16] F. Withers, M. Dubois, and A. K. Savchenko, Phys. Rev. B 82, 073403 (2010).

[17] Z. H. Ni, T. Yu, Y. H. Lu, Y. Y. Wang, Y. P. Feng, and Z. X. Shen, ACS Nano 2, 2301 (2008).

[18] J. H. Bardarson, M. Titov, and P. W. Brouwer, Phys. Rev. Lett. 102, 226803 (2009).

[19] R. R. Hartmann, N. J. Robinson, and M. E. Portnoi, Phys. Rev. B 81, 245431 (2010).

[20] C. A. Downing, D. A. Stone, and M. E. Portnoi, Phys. Rev. B 84, 155437 (2011).

[21] R. Asgari, M. I. Katsnelson, and M. Polini, Ann. Phys. (Berlin) 526, 359 (2014).

[22] Y. Wang, V. W. Brar, A. V. Shytov, Q. Wu, W. Regan, H. Z. Tsai, A. Zettl, L. S. Levitov, and M. F. Crommie, Nat. Phys. 8, 653 (2012).

[23] T. Ya. Tudorovskiy and A. V. Chaplik, JETP Lett. 84, 619 (2006).

[24] R. Jackiw, Phys. Scrita, T 146, 014005 (2012).

[25] C. Y. Hou, C. Chamon, and C. Mudry, Phys. Rev. Lett. 98, 186809 (2007).

[26] M. I. Katsnelson and K. S. Novoselov, Solid State Commun. 143, 3 (2007); M. I. Katsnelson and A. K. Geim, Philos. Trans. R. Soc. London A 366, 195 (2008).

[27] D. S. Novikov, Phys. Rev. B 76, 245435 (2007).

[28] F. Stern and W. E. Howard, Phys. Rev. 163, 816 (1967).

[29] F. Guinea, J. Low Temp. Phys. 153, 359 (2008).

[30] D. M. Basko, Phys. Rev. B 78, 115432 (2008).

[31] M. Titov, P. M. Ostrovsky, I. V. Gornyi, A. Schuessler, and A. D. Mirlin, Phys. Rev. Lett. 104, 076802 (2010).

[32] P. M. Ostrovsky, M. Titov, S. Bera, I. V. Gornyi, and A. D. Mirlin, Phys. Rev. Lett. 105, 266803 (2010). 
[33] T. O. Wehling, S. Yuan, A. I. Lichtenstein, A. K. Geim, and M. I. Katsnelson, Phys. Rev. Lett. 105, 056802 (2010).

[34] M. Schneider and P. W. Brouwer, Phys. Rev. B 84, 115440 (2011).

[35] L. A. Ponomarenko, R. Yang, T. M. Mohiuddin, M. I. Katsnelson, K. S. Novoselov, S. V. Morozov, A. A. Zhukov, F. Schedin, E. W. Hill, and A. K. Geim, Phys. Rev. Lett. 102, 206603 (2009).

[36] J. H. Chen, W. G. Cullen, C. Jang, M. S. Fuhrer, and E. D. Williams, Phys. Rev. Lett. 102, 236805 (2009).

[37] Z. H. Ni, L. A. Ponomarenko, R. R. Nair, R. Yang, S. Anissimova, I. V. Grigorieva, F. Schedin, Z. X. Shen, E. H. Hill, K. S. Novoselov, and A. K. Geim, Nano Lett. 10, 3868 (2010).

[38] M. Monteverde, C. Ojeda-Aristizabal, R. Weil, K. Bennaceur, M. Ferrier, S. Guéron, C. Glattli, H. Bouchiat, J. N. Fuchs, and D. L. Maslov, Phys. Rev. Lett. 104, 126801 (2010).

[39] A. Cresti, F. Ortmann, T. Louvet, D. Van Tuan, and S. Roche, Phys. Rev. Lett. 110, 196601 (2013).

[40] R. L. Heinisch, F. X. Bronold, and H. Fehske, Phys. Rev. B 87, 155409 (2013).

[41] J.-S. Wu and M. M. Fogler, Phys. Rev. B 90, 235402 (2014).

[42] V. V. Zalipaev, D. N. Maksimov, C. M. Linton, and F. V. Kusmartsev, Phys. Lett. A 377, 216 (2013).

[43] A. Deshpande and B. J. Le Roy, Physica E 44, 743 (2012).

[44] M. M. Fogler, D. S. Novikov, and B. I. Shklovskii, Phys. Rev. B 76, 233402 (2007).

[45] F. de Juan, A. Cortijo, and M. A. H. Vozmediano, Phys. Rev. B 76, 165409 (2007).

[46] K. Xu, P. Cao, and J. R. Heath, Nano Lett. 9, 4446 (2009).

[47] T. Mashoff, M. Pratzer, V. Geringer, T. J. Echtermeyer, M. C. Lemme, M. Liebmann, and M. Morgenstern, Nano Lett. 10, 461 (2010).

[48] N. N. Klimov, S. Jung, S. Zhu, T. Li, C. A. Wright, S. D. Solares, D. B. Newell, N. B. Zhitenev, and J. A. Stroscio, Science 336, 1557 (2012).

[49] D. Moldovan, M. Ramezani Masir, and F. M. Peeters, Phys. Rev. B 88, 035446 (2013).

[50] R. Carrillo-Bastos, D. Faria, A. Latge, F. Mireles, and N. Sandler, Phys. Rev. B 90, 041411(R) (2014).

[51] M. Schneider, D. Faria, S. Viola Kusminskiy, and N. Sandler, Phys. Rev. B 91, 161407(R) (2015).

[52] F. Calogero, Variable Phase Approach to Potential Scattering (Academic Press, New York, 1967).

[53] V. Babikov, Method of the Phase Functions in Quantum Mechanics (Nauka, Moscow, 1971).

[54] M. E. Portnoi, Pisma Zh. Tekh. Fiz. 14, 1252 (1988) [Sov. Tech. Phys. Lett. 14, 547 (1988)].

[55] M. E. Portnoi and I. Galbraith, Solid State Commun. 103, 325 (1997).

[56] M. E. Portnoi and I. Galbraith, Phys. Rev. B 58, 3963 (1998).

[57] M. E. Portnoi and I. Galbraith, Phys. Rev. B 60, 5570 (1999).

[58] H. Ouerdane, R. Varache, M. E. Portnoi, and I. Galbraith, Eur. Phys. J. B 65, 195 (2008).

[59] P. M. Morse and W. P. Allis, Phys. Rev. 44, 269 (1933).

[60] D. A. Stone, C. A. Downing, and M. E. Portnoi, Phys. Rev. B 86, 075464 (2012).
[61] D. S. Miserev, arXiv:1408.5611.

[62] N. Levinson, Mat. Fys. Medd. K. Dan. Vidensk. Selsk. 25, 3 (1949).

[63] R. G. Newton, Scattering Theory of Waves and Particles (Dover, Mineola, NY, 2002).

[64] J. Friedel, Philos. Mag. 43, 153 (1952).

[65] F. G. Fumi, Philos. Mag. 46, 1007 (1955).

[66] C. A. Downing, J. Math. Phys. 54, 072101 (2013).

[67] C. A. Downing, Open Physics 11, 977 (2013).

[68] A. De Martino, L. Dell'Anna, and R. Egger, Phys. Rev. Lett. 98, 066802 (2007).

[69] A. Zazunov, A. Kundu, A. Hütten, and R. Egger, Phys. Rev. B 82, 155431 (2010).

[70] M. Ramezani Masir, P. Vasilopoulos, and F. M. Peeters, J. Phys. Condens. Matter 23, 315301 (2011).

[71] M. Ramezani Masir, A. Matulis, and F. M. Peeters, Phys. Rev. B 84, 245413 (2011).

[72] S. Y. Zhou, G. H. Gweon, A. V. Fedorov, P. N. First, W. A. de Heer, D. H. Lee, F. Guinea, A. H. Castro Neto, and A. Lanzara, Nat. Mater. 6, 770 (2007).

[73] A. V. Rozhkov, G. Giavaras, Y. P. Bliokh, V. Freilikher, and F. Nori, Phys. Rep. 503, 77 (2011).

[74] This condition prohibits potentials decaying more slowly than $r^{-2}$ as $r \rightarrow \infty$. Otherwise, the asymptotic solution of Eq. (5) always adds to the phase shift however large $r$ becomes, as is familiar from the asymptotic behavior of the Coulomb wave function in the nonrelativistic case.

[75] M. Abramowitz and I. Stegun, Handbook of Mathematical Functions (Dover, Mineola, NY, 1972).

[76] C. W. J. Beenakker, Annu. Rev. Condens. Matter Phys. 4, 113 (2013).

[77] Q. Lin, Phys. Rev. A. 57, 3478 (1998).

[78] S.-H. Dong, X.-W. Hou, and Z.-Q. Ma, Phys. Rev. A 58, 2160 (1998).

[79] H.-Y. Chen, V. Apalkov, and T. Chakraborty, Phys. Rev. Lett. 98, 186803 (2007)

[80] In this estimate, we use $h_{2}=300 \mathrm{~nm}, h_{1}=200 \mathrm{~nm}, s=40 \mathrm{~nm}$, $R=5 \mathrm{~nm}$, and $n_{1}=n_{2}=2$.

[81] M. Zarenia, A. Chaves, G. A. Farias, and F. M. Peeters, Phys. Rev. B 84, 245403 (2011).

[82] M. Grujić, M. Zarenia, A. Chaves, M. Tadić, G. A. Farias, and F. M. Peeters, Phys. Rev. B 84, 205441 (2011).

[83] T. Stauber, N. M. R. Peres, and F. Guinea, Phys. Rev. B 76, 205423 (2007).

[84] G. Parzen, Phys. Rev. 80, 261 (1950).

[85] Y. Wang, D. Wong, A. V. Shytov, V. W. Brar, S. Choi, Q. Wu, H.-Z. Tsai, W. Regan, A. Zettl, R. K. Kawakami, S. G. Louie, L. S. Levitov, and M. F. Crommie, Science 340, 734 (2013).

[86] Y. Zhao, J. Wyrick, F. D. Natterer, J. F. Rodriguez-Nieva, C. Lewandowski, K. Watanabe, T. Taniguchi, L. S. Levitov, N. B. Zhitenev, and J. A. Stroscio, Science 348, 672 (2015).

[87] E. Margapoti, P. Strobel, M. M. Asmar, M. Seifert, J. Li, M. Sachsenhauser, O. Ceylan, C.-A. Palma, J. V. Barth, J. A. Garrido, A. Cattani-Scholz, S. E. Ulloa, and J. J. Finley, Nano Lett. 14, 6823 (2014). 